\title{
WORKFLOWS IN CONTENT MANAGEMENT SYSTEMS
}

\author{
Pedro Pico and Alberto Rodrigues da Silva \\ Instituto Superior Técnico, Universidade Técnica de Lisboa, Portugal \\ pedro.coelho.pico@gmail.com,alberto.silva@acm.org
}

\begin{abstract}
Keywords: Workflow, Automation, Content Management System (CMS), Workflow Management System (WMS).
Abstract: Workflow is a popular concept in nowadays. Organizations try to implement Workflows that are flexible and optimized, but the simple realization of Workflows inside an organization is not always a simple task. Thereby the perception of its definition and endeavours can bring several benefits. One tool that helps to structure and optimize organization's business processes are Content Management Systems, CMS. These applications provide a separation between content and services. When these two concepts meet in a CMS which supports Workflow, organizations may take advantage, becoming more agile, flexible and dynamic, while their tasks are automated. Ultimately these tools will help to increase the organization's productivity and establish the desired business processes.
\end{abstract}

\section{INTRODUCTION}

Workflow is an abstract concept that is currently relevant within Software Engineering as well as Organizational Engineering. Prove is the significant number of companies that is increasingly embracing it. Due to the richness and abstraction of the concept, Workflow is mentioned by Software Industry endeavors, meaning that it can be applied successful to Software application design and development. Bearing that in mind, the Workflow Management Coalition, WFMC http://www.wfmc.org is the reference organization responsible for the standard specification of Workflow engines and related specifications. The WFMC defines Workflow as "the computerized facilitation or automation of a business process, in whole or part"( David Hollingsworth, 1995). On the other hand, Marshak defines Workflow as "The automation of the processes we use every day to make our business through. A Workflow Application does automatically the sequence of actions, activities and tasks to run a process, including all the routing within the stages of each instance of a process, as well as the tools to manage the process itself", (R onni T. Marshak, 1995).

Automation and business processes are concepts that are mentioned on both definitions, leading to a first definition of Workflow as a "business process automation".
WFMC still introduces the concept of a Workflow Management System, as being a "system that completely defines, manages and executes the workflow through the execution of software whose order of execution is driven by a computer representation of the workflow logic", (David Hollingsworth, 1995).

These definitions indicate the Workflow logic has to be represented in a formal language, so that running software may be able to build, manage and execute that workflow logic.

On the other hand, Content Management Systems, CMS (are also known as ECMS (Enterprise Content Management Systems)), (James Robertson, 2002), are an emergent class of software systems that promotes the management of structured and unstructured business data (aka "content") of organizations, such as records of customers or links, as well as documents, pieces of text, images or videos. CMS provides the following benefits: extensibility, modularization and platform reuse; content and presentation independence; easy management and publication of contents; integrated support of multi-language contents; integrated support of users and access control management.

We identify in this paper some application scenarios where workflow technology can be applied with advantages. In particular, we discuss several issues concerning the use and adoption of workflows features into the domain of CMS platforms and respective CMS-based applications. 


\section{WORKFLOW'S APPLICATION SCENARIOS}

Workflow concepts and technologies can be applied into different application scenarios, such as (1) system integration support, (2) user interface and (3) content workflow in CMS (Content Management Systems).

Workflow Support in System's Integration. Information System's high-level abstractions and business service's interactions may be seen as a Workflow system, (Michael Rowell, 2001). Currently, Service Oriented Architecture (SOA), Dirk Krafzig, 2005, is an example of this approach, in which technology is only a tool for orchestration of business processes and services. According to the SOA approach there should be a Service repository which agglomerates services - which according to the OASIS organisation is defined as "a mechanism to enable access to one or more capabilities, where the access is provided using a prescribed interface and is exercised consistent with constraints and policies as specified by the service description.". These services, in their turn, communicate among them and with front and back-end applications, through a communication channel. To accomplish this, each service application must expose a programming or computing interface. Workflow plays the role of orchestrating the interactions amongst all these services. Microsoft Biztalk and BEA WebLogic are the leading application servers that support SOA in the industry.

Workflow Support in User Interface Software Applications. The Workflow concept can also be applied to the end-user interface definitions for software applications. The links between interaction spaces (e.g. web page, window screen) are events that may be triggered by human or third-party applications. For example, the submission of a web form is a human interaction, while the presentation of a RSS (Really Simple Syndication) feed is a thirdparty application interaction.

To make the most out of this vision of software design and implementation, some companies have been publishing Interface Workflow Managers. This functionality is usually associated with the software developing process since the tools that support these features are usually within the Integrated Developing Environments, IDE, of the existing platforms. With Microsoft Visual Studio, (Dino Esposito, 2002), the users have the ability to visually edit and see their application's interfaces as a tree of nodes, in which each node is an interaction space and the connection among nodes define the hierarchy of the nodes in the tree. On the other hand, Sun released in 2006 JAVA Web Studio Creator, (Gail Anderson, Paul Anderson, 2006), supporting the same functionality for J2EE applications.

Workflow Support in CMS. Content Management Environments are also a relevant application for Workflows. These Systems evolved from metaapplications and frameworks which were used to produce other applications. The urge for Documental Management Applications left only one step further what would later be known as Content Management Applications. The difference between these two is the object that is managed, while the former manage documents, the latter manage contents, which is an abstraction in which documents can be included.

In these environments a Workflow can be seen as the set of stages that content may assume since its creation until it is made available. Consider the following example: (1) a document is created and submitted to an application; (2) then it is approved in chain by a set of users; (3) until it becomes visible for all convenient users.

In this example the stage transitions are mainly triggered by human interaction. Users trigger stage transitions so that contents may evolve through the hierarchical structure of the organization, being progressively approved by users with more and more responsibilities. This type of Workflow systems has to make sure that if content is not approved by a user, it must return to its previous stage. Stage transitions also have to be able to trigger automatic actions. For instance, sending e-mails so users may be notified of pending decisions they have to make. Stage transitions may not only be triggered by human interaction, but also by third-party applications.

\section{CMS AND WORKFLOW}

Content Management Systems promote the clear separation between contents and services. The latter are responsible for content's presentation, manipulation and access, while the former are the artefacts that are passed throughout services. Pictures, texts, links, news, videos, and documents are all examples of contents.

The main goal of Workflows in CMS is to provide a path for contents since their creation, until 
they are made available for other users to see - often defined as publication. The path can be described by a set of consecutive evaluations, usually referred as stages, in which users, defined by a specific business role may, or may not, approve contents.

For example, in a newspaper, each article, after written by a journalist, must be reviewed by the journalist's supervisor. If the article is approved, it will go on to the next evaluation, and an upper supervisor will have to evaluate the article; otherwise it will return to the previous stage, and the journalist will have to rewrite the article. The evaluations will go on in chain until eventually some supervisor will approve the article's publication.

A Workflow in a CMS is exactly the chain of evaluations that content undergoes since its creation until its publication. It is now assumed a Workflow has several stages. While each stage is associated with one role - set of users - who are responsible for evaluating the content - either approving it, or declining it.

In the newspaper example, when a supervisor declines content, the content will return to the previous stage so it can be reviewed or rewritten again. If, on the other hand, the content is approved it will move on to the next stage. If it achieves the last stage, the content will be published. While in the physical world articles are printed in paper, in the digital world contents may either be created or replace existing versions of the content. As an example it can be considered any page available in the wikipedia web site, http://wikipedia.org. In this web site each page may be replaced by a new version, as well as new pages may be created. Pages are the contents in this example.

The multiplicity of instances of the same content that have to co-exist lead to the need of content versioning. This happens since at least two versions of the same content will be needed. One that is published, and thereby the one all users can see, and another one which is evaluated by supervisors, and thereby available for supervisors to see. The former will be referred as the published version, while the latter will be referred as the draft version. This can lead us to the conclusion that content is characterized by its version.

Finally, there is also another aspect to be added, the possible existence of predefined actions that may occur every time a stage is achieved or departed from. A simple notification to the author every time the content he submitted is approved by a supervisor is an example of what a predefined action can be. Figure 1 illustrates how the mentioned concepts are connected in a CMS Workflow Domain model.

\section{CONCLUSIONS}

The adoption of Workflow management systems is urging in the Software industry, allowing the automation of content's production and integration, This will lead to an optimization of the organisation's business processes.

CMS platforms with Workflow features show how organizations can better support their business processes, regarding agility and flexibility, since they provide a beneficial separation between contents and services, allowing the business users to manage both in an easy and independent way.

In the CMS domain a Workflow has several stages. While each stage has one responsible role and a set of predefined actions that may be triggered either when the content arrives or leaves the stage. Finally the content, which is the artefact that goes through the stages of the Workflow, has to be identified by a version, since in the most simplistic scenario at least two instances of the same content will have to co-exist. Figure 1 ilustrates the domain model of Workflow in CMS.

Workflows can be used in CMS is such scenarios as documental management, e-commerce and submission of articles. Documental management concentrates on documents as its contents. The life cycle of a document includes its creation, edition, configuration of its visibility, deletion, etc. All such operations can have associated Workflows that provide automatism and flexibility to these processes. E-commerce functionality can also take advantage of Workflow support. For instance in a purchase tracking mechanism, actions as the locking of an item, the payment checking mechanism, the client notification, and the notification of the warehouse which is supposed to send the item to the customer, can all be included in a Workflow which is responsible for the coordination of each of these steps. The last example of Workflow functionality in a CMS is the publishing of articles. In this Use Case scenario a user can create an article and submit it to a CMS. The CMS can then, by means of a Workflow, deliver the article to a supervisor, who will revise it and either accept it, publishing it, or declining it, sending feed-back for the user to improve his article. The described scenarios show the relevance and applicability of Workflows not only to business processes, but in CMS.

CMS that support Workflow provide their users a relevant advantage, since steps that that are repeated very often may be automated, saving time and allowing the users to concentrate on the most important part of their jobs: to focus on their business tasks creating business value for their organizations. 


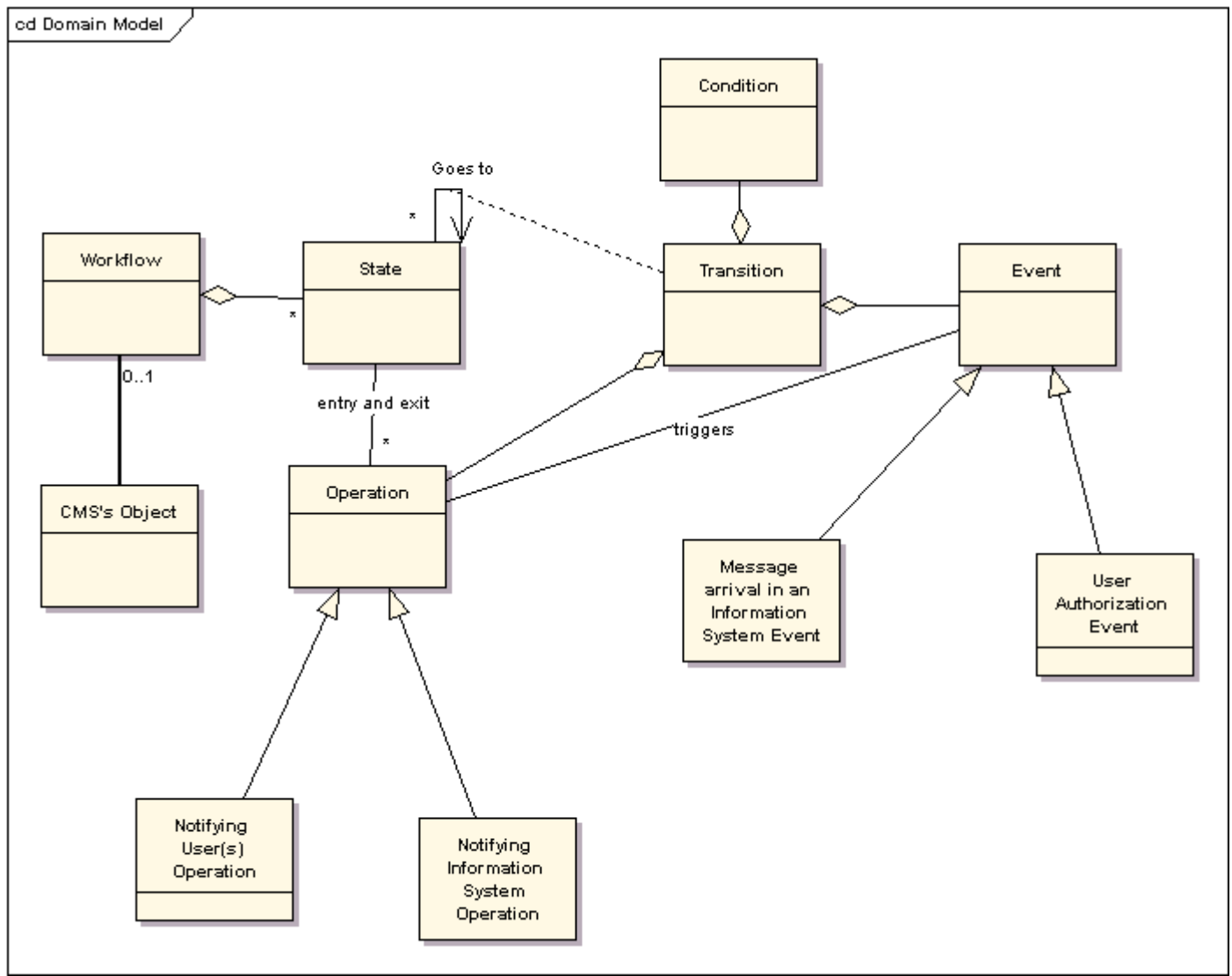

Figure 1: Main concepts of CMS's Workflow Support.

\section{REFERENCES}

David Hollingsworth, 1995. The Workflow Management Coalition Specification reference model.

Ronni T. Marshak, 1995. Workflow: applying automation to group processes, Groupware: technology and applications, Prentice Hall International (UK) Ltd., Hertfordshire, UK.

Michael Rowell, 2001. Understanding EAI: Enterprise Application Integration, Sams, Indianapolis, Indiana.

Dirk Krafzig, 2005. Karl Banke, Dirk Slama,. Enterprise SOA, Prentice Hall.

Dino Esposito, 2002. Building Web Solutions with ASP.NET and ADO.NET, Microsoft Press.

Gail Anderson, Paul Anderson, 2006. Java Studio Creator Field Guide, Sun Microsystems Press.

James Robertson, 2002. How to evaluate a content management system, KM Column. 